\title{
Plasma Current Sheath Motion in Coaxial Plasma Discharge
}

\author{
Tarek M. Allam, Hanaa A. El-Sayed, Hanaa M. Soliman \\ Plasma Physics and Nuclear Fusion Department, Nuclear Research Center, AEA, Cairo, Egypt \\ E-mail: hanaa.elshamy@yahoo.com \\ Received December 29, 2010; revised February 10, 2011; accepted March 1, 2011
}

\begin{abstract}
In this paper experiments and theoretical treatments [1] on $1.5 \mathrm{KJ}$ coaxial plasma discharge device have been carried out to show, plasma current sheath, PCS, motion in coaxial plasma discharge by studying: the effect of nitrogen gas pressure in the range from 1 to 2.2 Torr and the axial position of PCS along the coaxial electrodes on the modification factor, actual drive parameter, PCS curvature and shape (thickness). Also the dynamics of PCS along the coaxial electrodes due to the combination effect of induced azimuthal and axial magnetic fields induction has been detected experimentally by using a magnetic probe technique.
\end{abstract}

Keywords: Coaxial Discharge, Plasma Current Sheath, Drive Parameter

\section{Introduction}

Several studies [2-4] which are related to PCS formation in coaxial plasma discharge device is accomplished by two basic processes, 1) the formation of an axisymmetric current sheath at the surface of the insulator end of the coaxial electrodes breech, 2) axial acceleration of PCS by the electromagnetic force $\left(J_{r} \times B_{\theta}\right)$ along the annular of inter electrode discharge region.

An extensive study has been done by several authors in the field of axial PCS dynamics which depends on several macroscopic parameters like the energy of capacitor bank, the discharge current, the charging voltage and the curvature of the PCS [5-9]. Also a drive parameter $\left(I_{p} / a\right) / \sqrt{\rho}$, where $I_{p}$ is the peak discharge current, $a$ is the inner electrode radius and $\rho$ is the ambient gas density, has been derived previously to a plasma focus devices of different types [10-13]

This parameter determines the speed of the PCS in both axial and radial phases and it has a constant value for Mather type of different aspect ratios, gas pressures and discharge current [11]. Also some authors confirmed that the drive parameter has a remarkably constant value of PF devices with a range of energies from a few $\mathrm{KJ}$ to hundreds of KJ [14].

The goal of this paper extends to investigate the actual drive parameter of coaxial plasma discharge device as a function of PCS position during the axial rundown phase, also the behavior and shape of PCS are presented under a different discharge conditions.
This paper is contained the following sections, Section 2 describes the experimental setup, Section 3 presented the results and discussion. A conclusion of this work is presented in Section 4.

\section{Experimental Arrangment}

The coaxial plasma discharge device used in this work has five main parts, 1) the coaxial discharge chamber, 2) the energy storage system, 3) the electrical power supply, 4) the vacuum system and 5) the gas flow inlet system $[15,16]$.

A schematic diagram of the coaxial plasma discharge device and its electrical circuit are shown in Figures 1(a) and (b) respectively. The diameter of the inner and outer coaxial stainless steel electrodes are $2 a=5 \mathrm{~cm}$ and $2 b=$ $8.9 \mathrm{~cm}$ respectively. The inner electrode length is $13 \mathrm{~cm}$ and the inner electrode-to-outer electrode is $47 \mathrm{~cm}$. the inner and outer electrodes are insulated from each other by a tubular Perspex insulator of $1.5 \mathrm{~cm}$ length. The outer electrode muzzle has facilities for a magnetic probe tool.

The coaxial electrodes device capacitor bank is composed of three capacitors with total capacitance $30 \mu \mathrm{f}, 20$ $\mathrm{KV}$, in this work, energy stored in this bank is obtained by charging it to $10 \mathrm{KV}$ from a power supply. The capacitor bank transfers the energy of $1.5 \mathrm{KJ}$ to coaxial electrodes when switched through a spark gap switch which in turn switched by a $10 \mathrm{KV}$ triggering pulse. The device is filled with nitrogen gas with pressure varying 


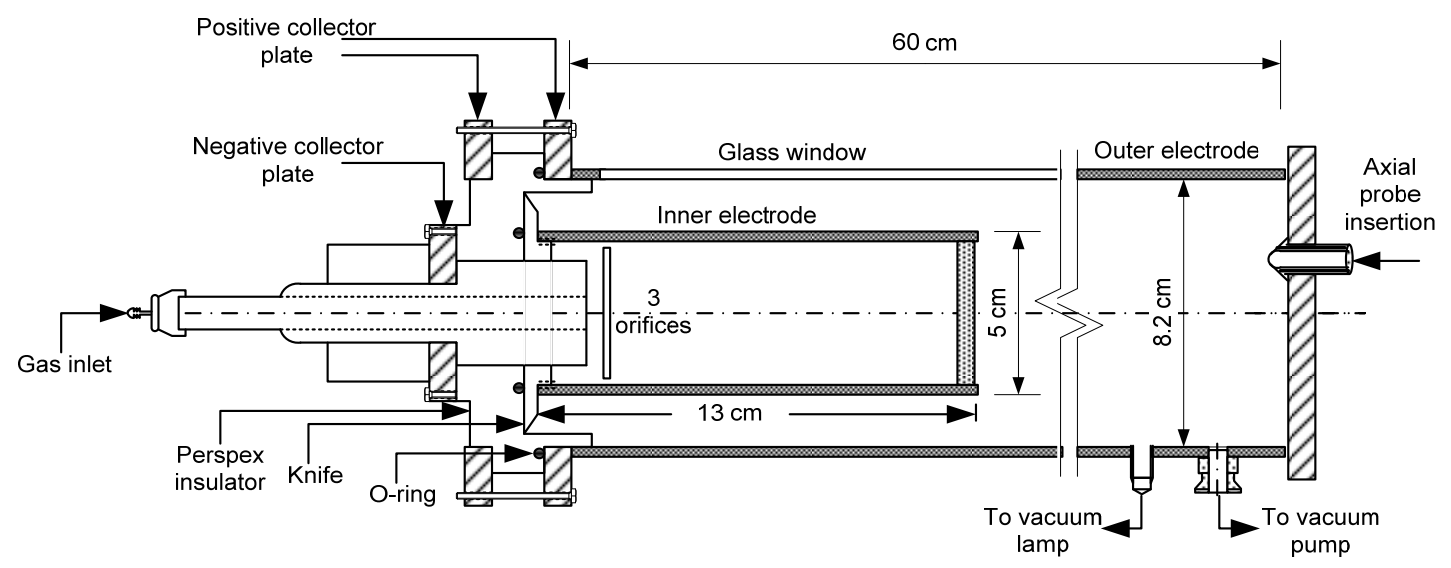

(a)

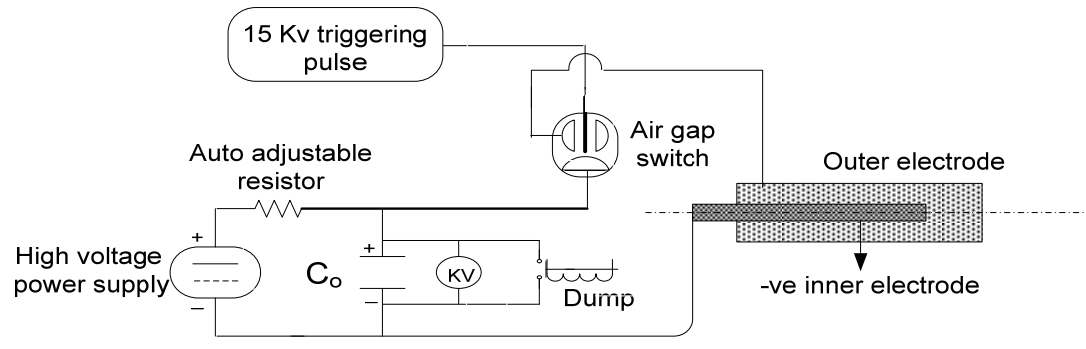

(b)

Figure 1. (a) Coaxial plasma discharge device; (b) Electrical circuit of the coaxial plasma discharge device.

from 1 to 2.2 Torr.

The data of experimental works were taken from an average of approximately from 5 to 7 shots for each gas pressures and axial distances under consideration.

\section{Results and Discussion}

In the present work the experimental and theoretical results of PCS motion are carried out along the axial distance and at the annular space between the two coaxial electrodes system, at radial distance, $r=$ average of inner, a and outer radius, $b$ of coaxial electrodes $=3.475 \mathrm{~cm}$.

Figure 2 shows the variation of the axial PCS velocity measured by a magnetic probe technique, $V_{a}$, with axial distance along the annular space between the coaxial electrodes and at $r=3.475 \mathrm{~cm}$ for different nitrogen gas pressures. It can be seen from this figure that the sheath velocity has their minimum value at the beginning of the discharge and then it increases gradually in axial phase to reach its maximum value nearly at the muzzle for all values of gas pressure under consideration. This behavior was attributed to the current density of plasma sheath, the gas mass swept by PCS along the coaxial electrodes and the mean free path. The theoretical axial velocity, $U_{a}$ calculated by using a snow-plough model [17] is given by,

$$
U_{a}=\left[\frac{\mu \ln (b / a)}{4 \pi^{2}\left(\frac{b^{2}}{a^{2}}-1\right)}\right]^{\frac{1}{2}} \frac{I_{p}}{\sqrt[a]{\rho}}
$$

A modification factor, $F=f_{c} / \sqrt{f_{m}}$ (where, $f_{m}$ is some percent of mass was sweeping by PCS and $f_{c}$ is some percent of bank current was driving the PCS) can be estimated from ratio of the measured axial PCS velocity, $V_{a}$ and the theoretical axial velocity, $U_{a}[17]$.

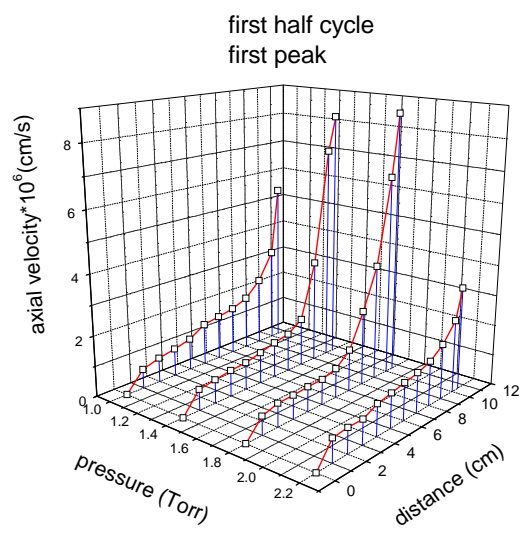

Figure 2. Variation of axial sheath velocity with respect to axial distance and gas pressure. 


$$
F=\frac{V_{a}}{U_{a}}
$$

Figure 3(a)-(d) show the variation of modification factor, $F$ and the axial distance, $Z$ (from breech to muzzle of coaxial electrodes system) at different nitrogen gas pressures $1,1.4,1.8,2.2$ torr. It can be seen from this figure, that for all values of gas pressures the distribution of $\mathrm{F}$ with $Z$ has approximately the same behavior and at a distances approach the coaxial muzzle, $F$ increases sharply to reach a maximum value.

Actual drive parameter, $D$ is estimated by multiplying $F$ with drive parameter $=I_{p} / \sqrt[a]{\rho}$,

$$
\left(D_{\text {actual }}=F \frac{I_{p}}{\sqrt[a]{\rho}}\right)
$$

In our case, $I_{p} \approx 54.5 \mathrm{KA}, \rho=1.48 \times 10^{-3}, 2.07 \times 10^{-3}$, $2.66 \times 10^{-3}$ and $3.26 \times 10^{-3} \mathrm{~kg} / \mathrm{m}^{3}$ at $P=1,1.4,1.8,2.2$ torr respectively and $a=2.5 \mathrm{~cm}$.

Figures 4(a)-(d) show the variation of $\ln (D)$ with axial distance $\ln (Z)$ at different gas pressures, this figure

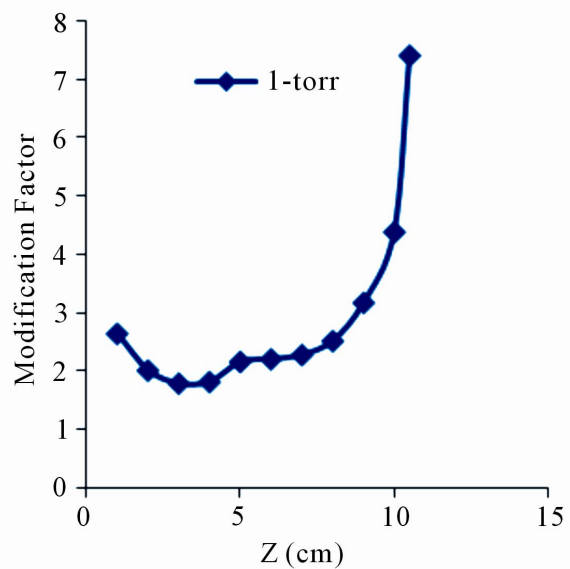

(a)

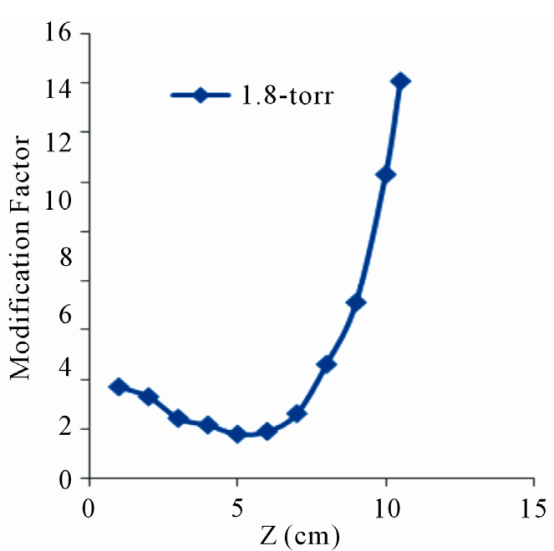

(c) indicates that $D$ is decreased from the breech to a distance approaches to approximately a mid-distance of coaxial electrodes length with different rates as follows:

$D \propto Z^{-0.346}$ for $Z$ varied from $(1$ to $4 \mathrm{~cm}$ ) for $P=1$ Torr.

$D \propto Z^{-0.38}$ for $Z$ varied from $(1$ to $4 \mathrm{~cm})$ for $P=1.4$ Torr.

$D \propto Z^{-0.45}$ for $Z$ varied from ( 1 to $4.7 \mathrm{~cm}$ ) for $P=1.8$ Torr.

$D \propto Z^{-0.557}$ for $Z$ varied from $(1$ to $4 \mathrm{~cm}$ ) for $P=2.2$ Torr.

After this distance, $D$ is increased in a different two regions with different rates as follows:

$D \propto Z^{0.52}$ for $Z$ varied from (4 to $8 \mathrm{~cm}$ ), and $D \propto Z^{3.06}$ for $Z$ varied from ( $8 \mathrm{~cm}$ to $\sim$ muzzle) for $P=$ 1 Torr.

$D \propto Z^{0.47}$ for $Z$ varied from (4 to $8 \mathrm{~cm}$ ), and $D \propto Z^{6.5}$ for $Z$ varied from ( $8 \mathrm{~cm}$ to $\sim$ muzzle) for $P=1.4$ Torr.

$D \propto Z^{0.45}$ for $Z$ varied from $(4.7$ to $7 \mathrm{~cm})$ and $D \propto Z^{4.44}$ for $Z$ varied from $(7 \mathrm{~cm}$ to $\sim$ muzzle $)$ for $P=$ 1.8 Torr.

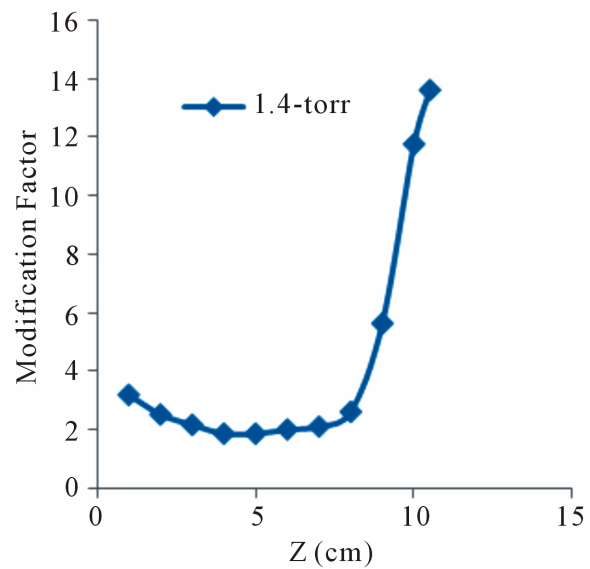

(b)

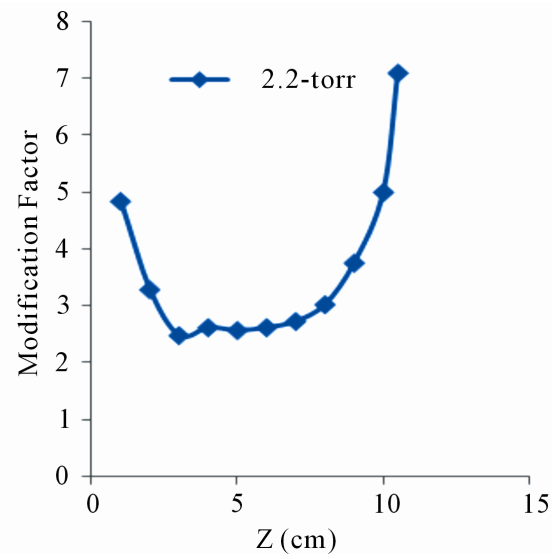

(d)

Figure 3. (a)-(d) Variation of modification factor, $F$, versus axial distance, $Z$. 


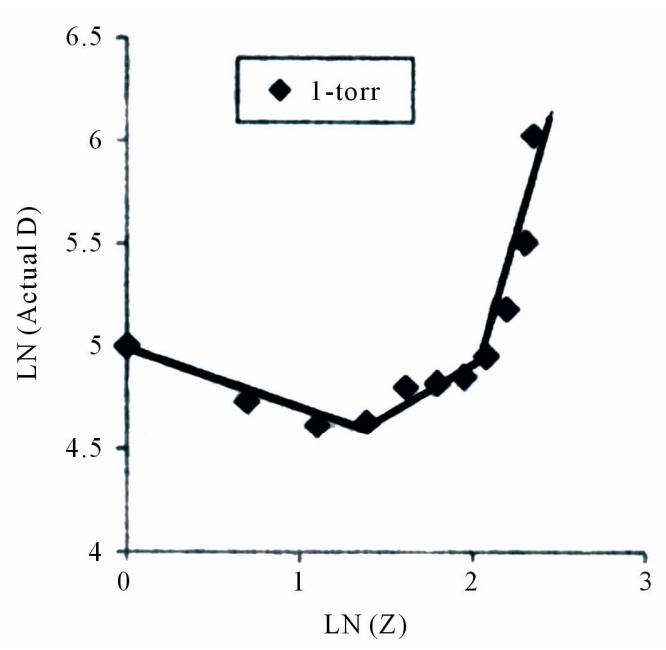

(a)

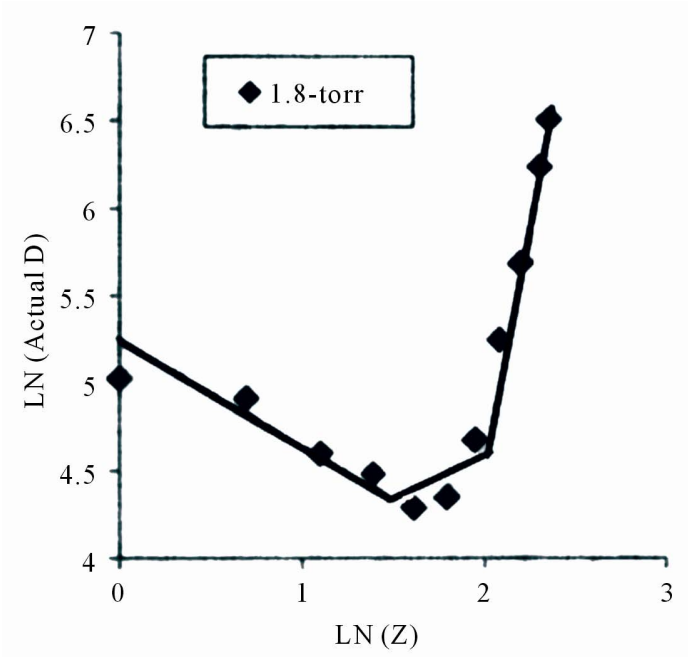

(c)

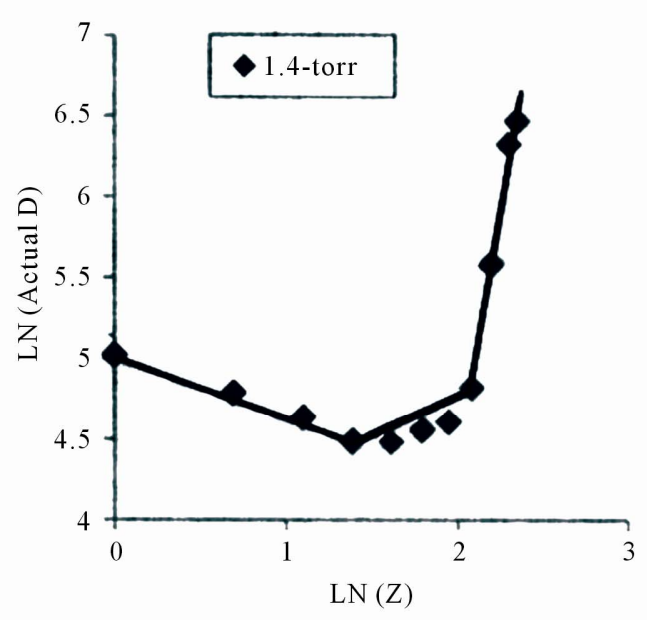

(b)

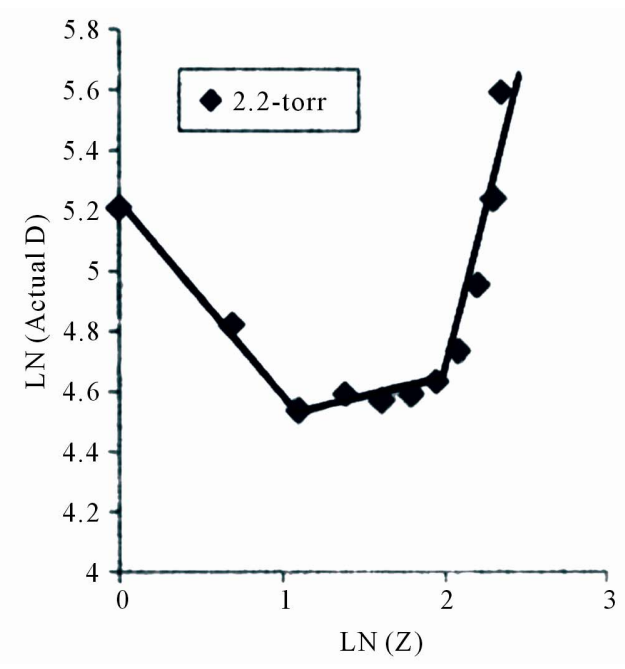

(d)

Figure 4. (a)-(d) Variation of $\ln ($ actual drive parameter, $D)$ versus $\ln (Z)$ at different gas pressures.

$D \propto Z^{0.247}$ for $Z$ varied from (4 to $7 \mathrm{~cm}$ ), and $D \propto Z^{2.285}$ for $Z$ varied from $(7 \mathrm{~cm}$ to $\sim$ muzzle) for $P=$ 2.2 Torr.

The above results illustrate that the rate of decreasing of $D$ with $Z$ is increased with increasing of gas pressure at axial distance, $Z$ from breech to $\sim 4 \mathrm{~cm}$, while at a mid-distance from $\sim 4 \mathrm{~cm}$ to $\sim 8 \mathrm{~cm}$, the rate of increasing of $D$ with $Z$ is decreased with increasing of gas pressure, finally at axial distance approaches to coaxial muzzle ( 8 $\mathrm{cm}$ to muzzle), the rate of increasing of $D$ with $Z$ as a function of gas pressure has a maximum value at $P=1.4$ torr.

In general $D$, for all values of gas pressure has a maximum value at a distance closes to coaxial electrodes muzzle.

The PCS thickness, $\lambda$ variations with axial distance, $Z$ as a function of gas pressures are estimated from a radial
PCS density $J_{r}$ data, $J_{r}=\frac{I(t)}{2 \pi r \lambda}$, where $I(t)$ is the discharge current, taking $I(t)=I_{p} \sin \sin w t$, $w=\frac{2 \pi}{\tau}=0.21 \times 10^{6} \mathrm{sec}^{-1}$.

Variation of $\ln$ (thickness of PCS, $\lambda$ in arbitrary unit) and $\ln (Z)$ is presented in Figures 5(a)-(d) at different gas pressures. This figure reveals that, $\lambda$ is increased from a distance closes to coaxial breech until a distance $\sim 8 \mathrm{~cm}$, then it decreased to reach a coaxial muzzle, with different rates as follows:

$\lambda \propto Z^{2.1}(1.6 \rightarrow 8 \mathrm{~cm}), \lambda \propto Z^{-2.9} \quad(8 \mathrm{~cm}$ to muzzle $)$ for $P=1$ Torr.

$\lambda \propto Z^{2}(1.6 \rightarrow 8 \mathrm{~cm}), \lambda \propto Z^{-3} \quad(8 \mathrm{~cm}$ to muzzle $)$ for $P=1.4$ Torr.

$\lambda \propto Z^{1.166} \quad(1.6 \rightarrow 8 \mathrm{~cm}), \lambda \propto Z^{-5.5} \quad(8 \mathrm{~cm}$ to muzzle $)$ 


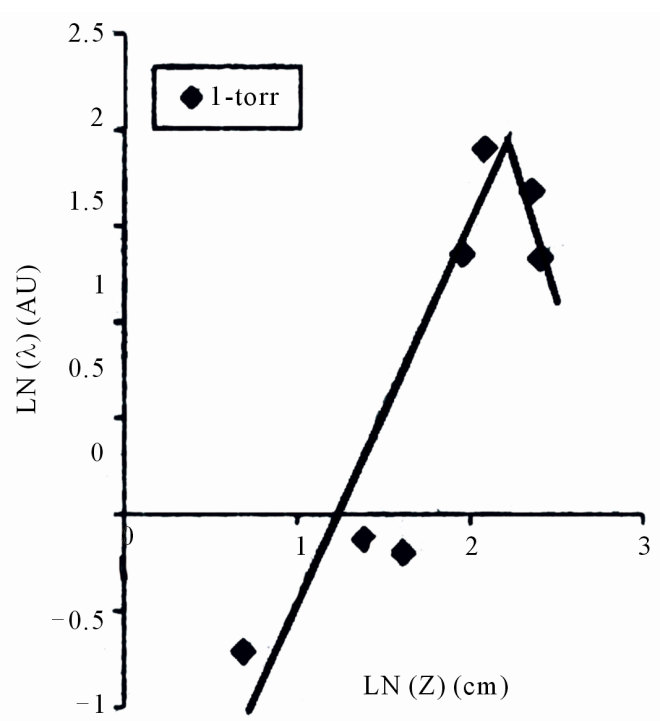

(a)

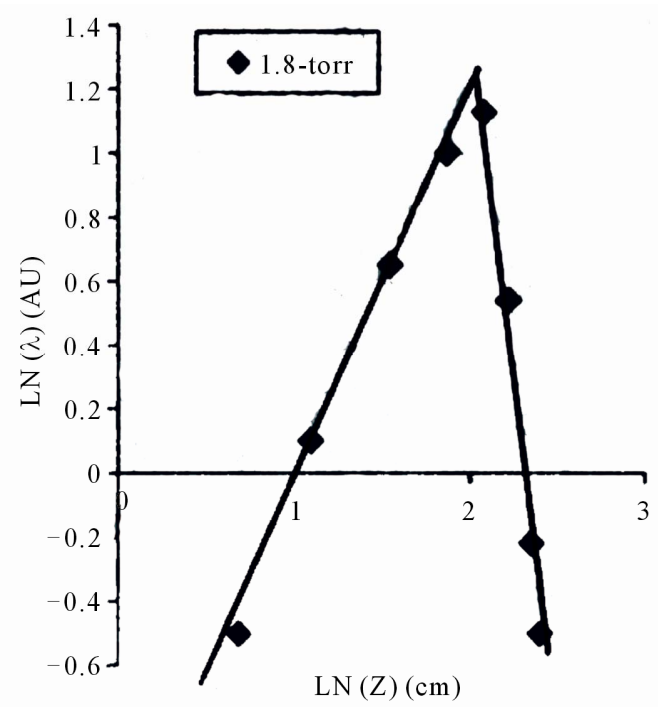

(c)

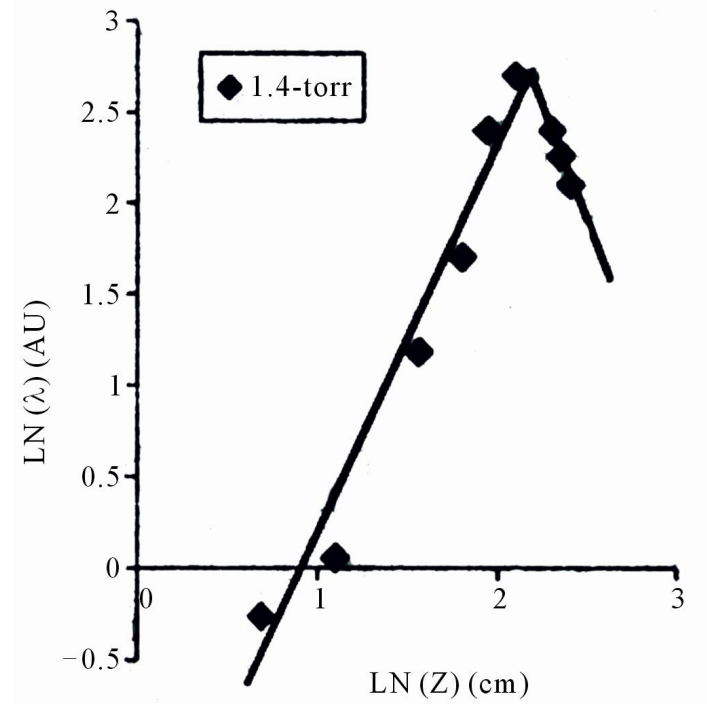

(b)

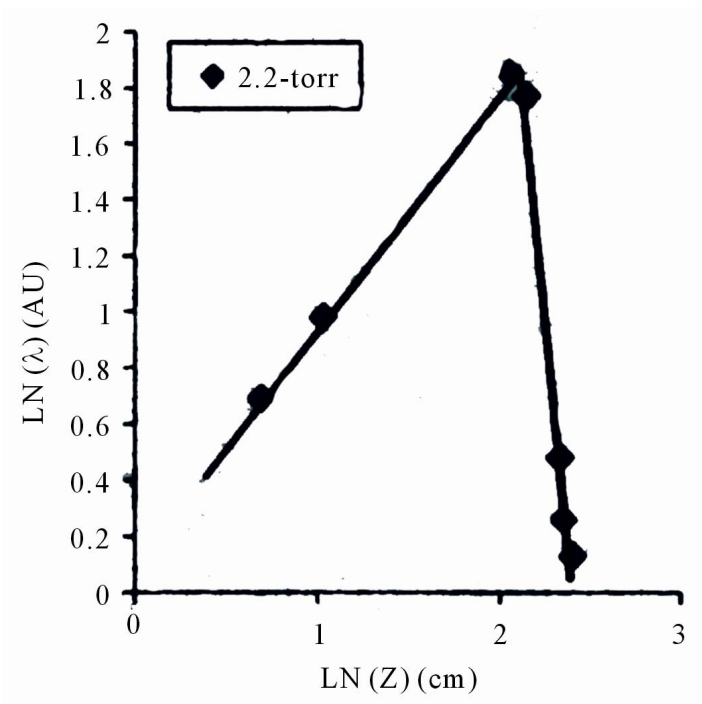

(d)

Figure 5. (a)-(d) The relation between $\ln (\lambda)$ and $\ln (Z)$ at different gas pressures.

for $P=1.8$ Torr.

$\lambda \propto Z^{0.85} \quad(1.6 \rightarrow 8 \mathrm{~cm}), \quad \lambda \propto Z^{-5.44} \quad(8 \mathrm{~cm}$ to muzzle $)$ for $P=2.2$ Torr.

These results clear that, the rate of increasing of $\lambda$ with $\mathrm{Z}$ is decreased with increasing of gas pressure from a distance closes to coaxial breech until $Z=8 \mathrm{~cm}$, and at a distances varied from $8 \mathrm{~cm}$ to muzzle; the rate of decreasing of $\lambda$ with $Z$ is increased with increasing of most values of gas pressures and it has a maximum value at $P$ $=1.8$ torr. Previous results indicate that, a decrease in thickness of PCS after a propagating an axial distance of $8 \mathrm{~cm}$, is clearly reflected the mass loss effects [17].

Inclination angle $\theta$ of PCS with $Z$-axis along the coaxial electrodes can be detected from the determination of axial distance traveled by PCS at a distance closes to inner surface of outer electrode and at radial distance $r=$ $3.475 \mathrm{~cm}$ during the same time. Variation of inclination angle $\theta$ of PCS with $Z$-axis along the coaxial electrodes and at different values of gas pressure is cleared in Figures 6(a)-(d). It can be seen from this figure that $\theta$ is damped with two regions, the first one from the breech until a distance $Z \sim 4 \mathrm{~cm}$ and the second from $Z \sim 4 \mathrm{~cm}$ to muzzle with different rates as follows:

$\theta \propto Z^{-0.19}(0.5$ to $4 \mathrm{~cm})$ and $\theta \propto Z^{-0.7} \quad(4 \mathrm{~cm}$ to muzzle) for $P=1$ Torr.

$\theta \propto Z^{-0.185}(0.55$ to $4 \mathrm{~cm})$ and $\theta \propto Z^{-0.773} \quad(4 \mathrm{~cm}$ to muzzle) for $P=1.4$ Torr.

$\theta \propto Z^{-0.22}(0.75$ to $4 \mathrm{~cm})$ and $\theta \propto Z^{-0.68} \quad(4 \mathrm{~cm}$ to 


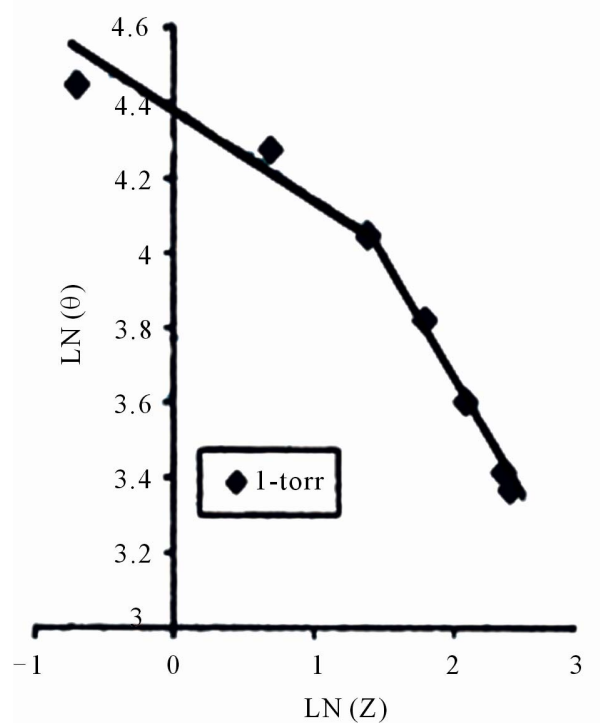

(a)

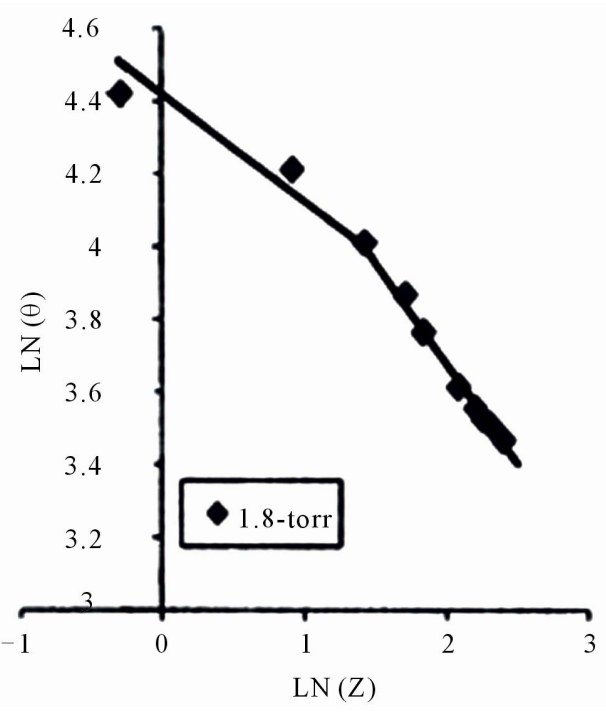

(c)

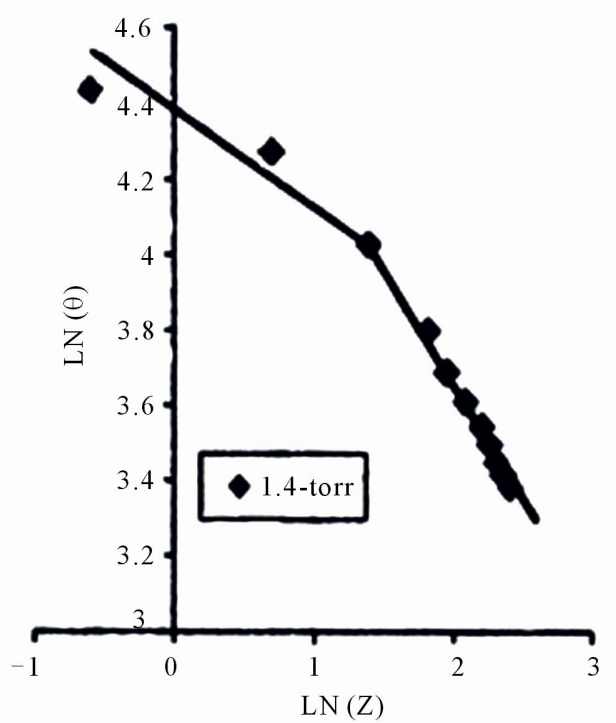

(b)

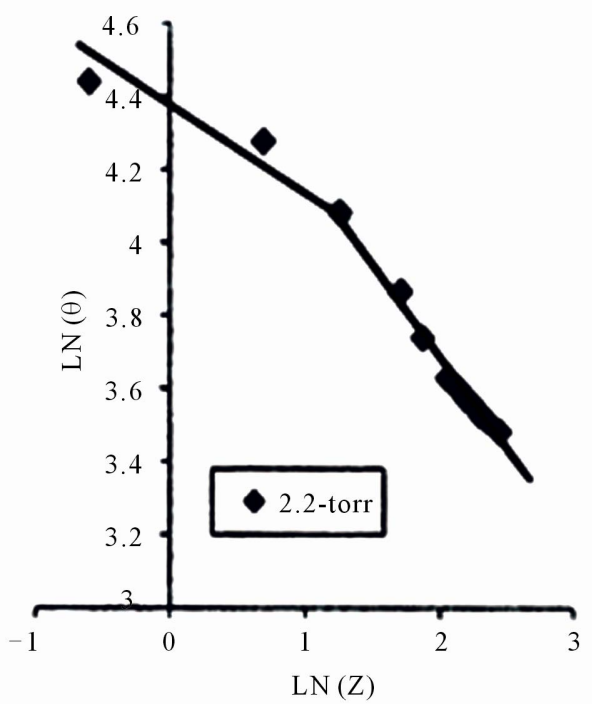

(d)

Figure 6. (a)-(d) Variation of $\ln (\theta)$ with $\ln (Z)$ at different gas pressures.

muzzle) for $P=1.8$ Torr.

$\theta \propto Z^{-0.2}(0.55$ to $4 \mathrm{~cm})$ and $\theta \propto Z^{-0.58} \quad(4 \mathrm{~cm}$ to muzzle) for $P=2.2$ Torr.

The above data show that, the rate of decreasing of angle $\theta$ with $Z$ has approximately the same values for all gas pressures under consideration from the breech to $Z=$ $4 \mathrm{~cm}$, while at axial distance from $4 \mathrm{~cm}$ to muzzle, the rate of decreasing of $\theta$ with axial distance $Z$ has a maximum value at $P=1.4$ torr i.e. at this pressure the parabolic PCS profile is more canted than other gas pressure values under consideration, also this behavior could be owing to the fact that the magnetic pressure $B^{2} / 2 \mu$ [3] has a peak value at this gas pressure value.
Variation of the ratio of induced axial and azimuthal magnetic fields $B_{z}$ and $B_{\theta}$ respectively, with the axial distance, $Z$ is shown in Figures 7(a)-(d) for different gas pressures. In general this figure demonstrated that $B_{z} / B_{\theta}$ varies between 0.75 and 4.5 , hence the magnetic helicity plays a role in the PCS motion which must be taken into account.

\section{Conclusions}

Experimental and theoretical results showed that a rapid increase of modification factor, $F=f_{c} / \sqrt{f_{m}}$ with axial distances, $Z$ approach to coaxial electrodes muzzle for all 


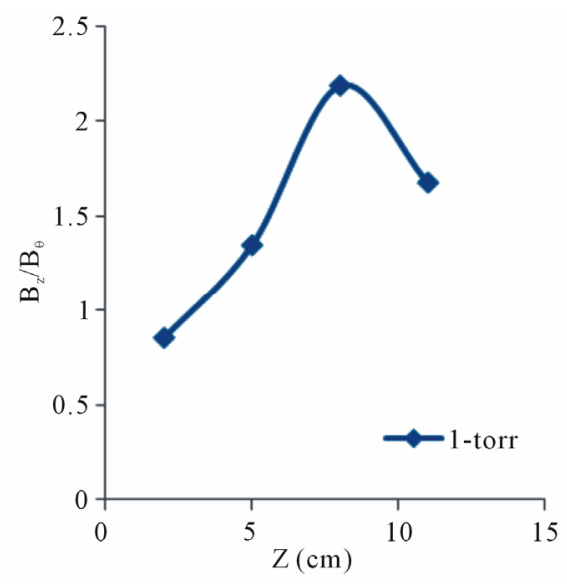

(a)

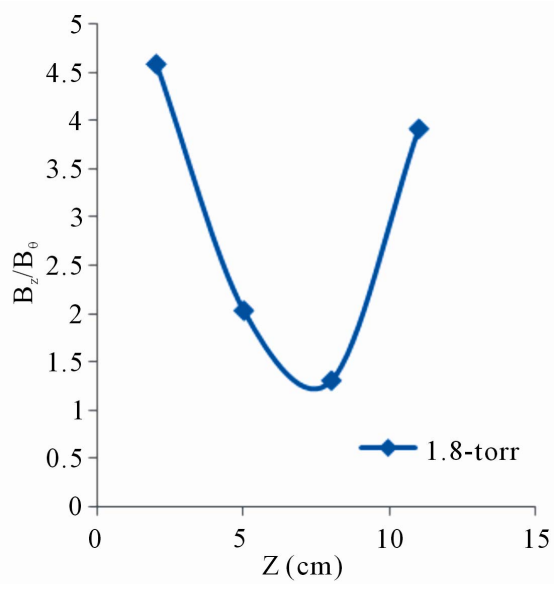

(c)

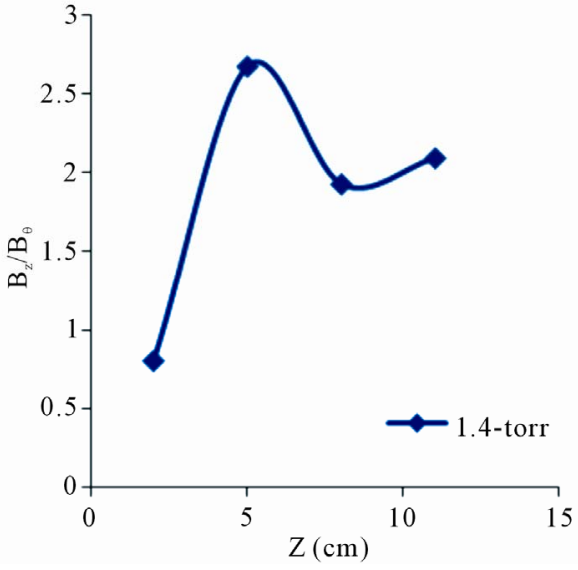

(b)

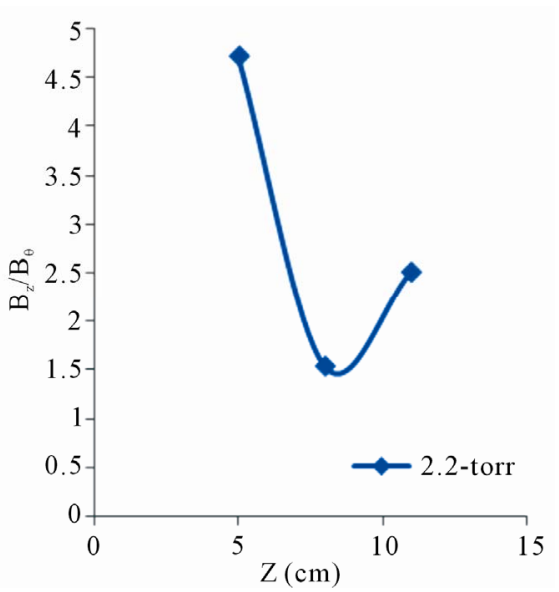

(d)

Figure 7. (a)-(d) Ratio of $B_{z} / B_{\theta}$ versus axial distance $Z$.

nitrogen gas pressures under consideration, this behavior suggests that, the current and mass shedding effect which taking place during the PCS motion along the coaxial electrodes is significant along this distance.

Results of actual drive parameter, $D$ or speed parameter, inclination angle, $\theta$ and thickness, $\lambda$ of PCS distribution with axial distance along the coaxial electrodes, $Z$ at different gas pressures demonstrated that, each of these parameters has two or three different regions with different rates, specially at a distance closes to coaxial muzzle. Results of axial distribution of $D, \theta, \lambda$ demonstrated that, these distributions affect by two important parameters namely the current shedding, $F_{c}$ and mass losses, $F_{m}$. The peak value of rate of change of the above parameters with axial distance, $Z$ is detected approximately at the gas pressure of 1.4 torr. A general view of PCS motion along the coaxial electrodes at different gas pressures is illustrated from the axial distribution of the ratio $B_{z} / B_{\theta}$, it showed that, the PCS has a helical structure along the coaxial electrodes. At a distance be- yond the coaxial muzzle (from $8 \mathrm{~cm} \sim$ muzzle) and at $P$ $=1.4$ torr, the PCS moves with approximately less helical motion than other gas pressures under consideration, i.e. the axial force $J_{r} \times B_{\theta}$ affecting on PCS motion is greater than the azimuthal force $J_{r} \times B_{z}$.

From the obtained results, one can conclude that, the proper PCS motion is found at approximately $P=1.4$ torr filling nitrogen gas pressure.

\section{References}

[1] S. Lee, B. C. Ton, C. S. Wong and A. C. Chew, "Technolgy of the Plasma Focus," Laser and Plasma Technology, World Scientific Publisher, Singapore City, 1985, pp. 387-420.

[2] S. Lee, "A Sequential Plasma Focus," IEEE Transactions on Plasma Science, Vol. 19, No. 5, 1991, pp. 912-919. doi:10.1109/27.108433

[3] S. P. Chew, S. Lee and B. C. Tan, "Current Sheath Studies in a $\mathrm{Co}=$ axial Plasma Focus Gun," Journal of Plasma 
Physics, Vol. 8, No. 1, 1972, pp. 21-31. doi: $10.1017 / \mathrm{S} 0022377800006905$

[4] J. N. Feugas, "The Influence of the Insulator Surface in the Plasma Behavior," Journal of Applied Physics, Vol. 66, No. 8, 1989, pp. 3467-3471. doi:10.1063/1.344102

[5] M. Zakaullah, G. Murtaza, I. Ahmed, F. N. Beg, M. M. Beg and M. Shabbir, "Comparative Study of Low Energy Mather-Type Plasma Focus Device," Plasma Sources Science and Technology, Vol. 4, No. 1, 1995, pp. 117124. doi:10.1088/0963-0252/4/1/012

[6] F. N. Beg, M. Zakaullah, M. Nisar and G. Murtaza, "Role of Anode Length in a Mother type Plasma Focus," Modern Physics Letters B, Vol. 6, No. 10, 1992, pp. 593-597. doi:10.1142/S0217984992000685

[7] R. K. Rout, A. B. Garg, A. Shyam and M. Srinivasan, "Influence of Electrode and Insulator Materials on the Neutron Emission in a Low Energy Plasma Focus Device," IEEE Transactions on Plasma Science, Vol. 23, No. 6, 1995, pp. 996-1000. doi:10.1109/27.476488

[8] H. P. Randy, S. S. Loke, P. Lee, R. S. Rawat and S. Lee "Effects of Insulator Sleeve Length on Neutron and X-Ray Emissions from Deuterium Filled Dense Plasma Focus Device," 30th European Physics Society Conference Controlled Fusion and Plasma Physics, Vol. 27A, 2003, pp. 1-208. doi:10.1088/0963-0252/12/3/320

[9] M. Zakaullah, A. Waheed, S. Ahmed, S. Zeb and S. Hussain, "Study of Neutron Emission in a Low Energy Plasma Focus with $\beta$-Source Assisted Breakdown," Plasma Sources Science and Technology, Vol. 12, No. 3, 2003, pp. 443-448.

[10] T. Zheng, R. S. Rawat, S. M. Hassan and J. J. Lin, "Drive Parameter as a Design Consideration for Mother and Filippov Types of Plasma Focus," IEEE Transactions on
Plasma Science, Vol. 34, No. 5, 2006, pp. 2356-2362. doi:10.1109/TPS.2006.883390

[11] S. Lee and A. Serban, "Dimensions and Lifetime of the Plasma Focus Pinch," IEEE Transactions on Plasma Science, Vol. 24, No. 3, 1996, pp. 1101-1105. doi:10.1109/27.533118

[12] J. M. Koh, R. S. Rawat, A. Patran, T. Zhang, D. Wong, S. Springham, T. L. Tan, S. Lee and P. Lee, "Optimization of the High Pressure Operation Regime in a Plasma Focuse Device," Plasma Sources Science and Technology, Vol. 14, No. 1, 2005, pp. 12-18. doi:10.1088/0963-0252/14/1/002

[13] M. Scholz, R. Miklaszewski, M. Paduch, M. J. Sadowski, A. Szydlowski and K. Tomaszewski, "Preliminary Neutron Experiments with the PF-1000 Plasma Focus Facility," IEEE Transactions on Plasma Science, Vol. 30, No. 2, 2002, pp. 476-481. doi:10.1109/TPS.2002.1024279

[14] L. Soto, "New Trends and Future Perspectives on Plasma Focus Research," Lasma Physics and Controlled Fusion, Vol. 47, No. 5A, 2005, pp. A361-A381.

[15] H. A. El-Sayed, "Coaxial Plasma Discharge Dynamics and Characteristics," Ph. D. Thesis, Faculty of Engineering, Cairo University, Cario, 2009.

[16] H. M. Hussien, T. M. Allam, H. A. El-Sayed and H. M. Soliman, "Characterization of $1.5 \mathrm{KJ}$ Coaxial Plasma Discharge," Journal of Engineering and Applied Sciences, Vol. 56, No. 4, 2009, pp. 315-329.

[17] H. R. Yousefi, G. R. Etaati and M. Gharannevis, "Insulator Length Effects in Mother-Type Plasma Focus Device," 31st European Physical Society Conference on Plasma Physics, London, Vol. 28G, 28 June-2 July 2004, pp. 5006-5009. 